The Open Civil Engineering Journal
CrossMark

REVIEW ARTICLE

\title{
Discussion of Visual Technique for Seepage Experiment Based on Transparent Rock-Soil Material
}

\author{
Jianjun Liu ${ }^{1,2}$, Yao Wang, ${ }^{2,}$, Rui Song ${ }^{2}$ and Yue Sun ${ }^{2}$

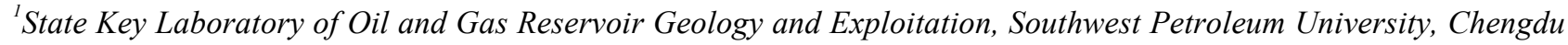 \\ 610500, China; \\ ${ }^{2}$ School of Geoscience and Technology, Southwest Petroleum University, Chengdu 610500, China;
}

Received: December 25, 2016

Revised: January 09, 2017

Accepted: March 07, 2017

\begin{abstract}
:
Introduction:

Due to the fact that the model experiment can simulate the flow process and movement law of fluid during the seepage experiment, seepage model experiment was widely applied to study the hydraulic characteristics of rock-soil mass and transportation of ground water and pollutants. While due to the opacity of the model medium in traditional seepage experiment, the flow process, diffusion law and occurrence state of fluid can not be observed intuitively during the experiment.
\end{abstract}

\section{Method:}

A new technique of seepage model experiment based on transparent rock-soil material (TRSM) was introduced in this paper, combined with optical observation method, digital imaging process technology and tracer technique, so as to realize the visual observation of seepage process.

\section{Results:}

The advantages and disadvantages compared with the traditional experiment were analyzed and the theoretical foundation for carrying out the seepage experiment based on TRSM was established.

Keywords: Seepage Mechanics, TRSM, Model experiment, Visualization, Optical observation, Digital image process.

\section{INTRODUCTION}

The seepage mechanics is a subject to study the flow law of the fluid (including gas and liquid) in porous media. It is not only an important branch of fluid mechanics, but also an interdisciplinary subject crossing with many disciplines such as rock mechanics, porous media physics and physical chemistry etc [1 - 3]. Since 1856, the French engineer Darcy put forward the liner seepage law, after years' development, the seepage mechanics made the considerable progress, and has been widely applied in many fields such as water conservancy, transportation, energy and biological etc $[4,5]$.

As one of the important methods in the study of seepage mechanic, seepage experiment has significance in the study of fluid flow. Due to the opacity of the model medium in traditional seepage experiment, the specific flow process, the flow and diffusion law, and the occurrence state of fluid can not be observed directly and clearly, therefore it can't form an intuitive and qualitative understanding of the seepage process during the experiment.

These existence limitations in traditional seepage experiment greatly restricted the further development of seepage

* Address correspondence to this author at the School of Geoscience and Technology, Southwest Petroleum University, Chengdu 610500, China; Tel: +86 028 83035158; Fax: +86 028 83035158; E-mail:542385778@qq.com 
mechanics. In order to solve these limitations and deepen understanding of seepage theory, carry out the visualization research of seepage experiment has great significance. As a consequence a new kind of seepage experiment method based on TRSM, combined with tracer technique, optical observation method and digital imaging process was introduced in this paper. Based on the method, the process of fluid flow in the model medium can be monitored and recorded clearly during the experiment. At the same time, the diffusion law and occurrence state of fluid in medium can also be recorded intuitively, thus realize the visual observation of seepage experiment. With the development of this kind of new experiment method, more and more model experiment can be carried out based on the new material and related experiment technique, such as water-flooding and hydrofracturing etc. This method has the advantages of low cost, simple device and easy operation, so it has a great application prospect in experimental operation and theoretical research of seepage mechanics.

\section{Traditional Seepage Experiment}

Through lots of experimental verification, Darcy established the seepage law of water in sand layer and the initial model of seepage experiment. And the experimental device showed in Fig. (1). Darcy's law can be described as the flow of homogenous fluid in porous media [6], and it is the basic law in seepage mechanics which can be expressed as:

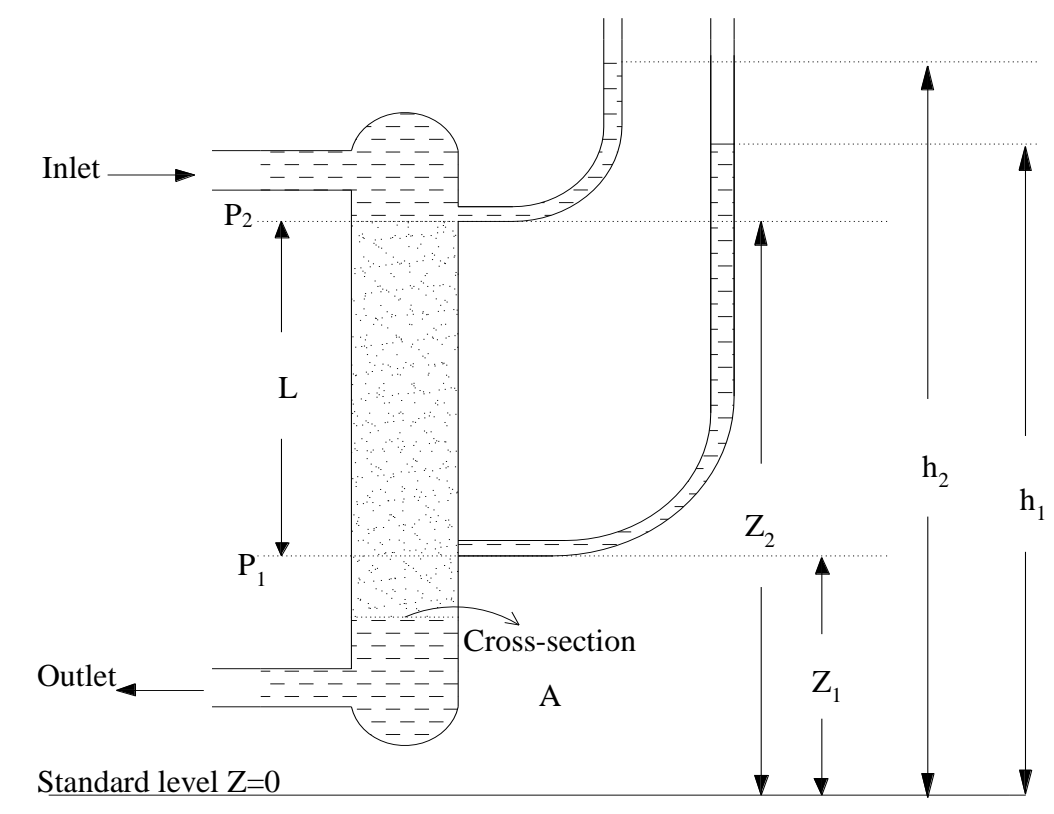

Fig. (1). Experimental device of Darcy seepage.

$$
\begin{gathered}
Q=\mathrm{kA} \frac{h_{2}-h_{1}}{L} \\
\mathrm{i}=\frac{h_{2}-h_{1}}{L}
\end{gathered}
$$

Where $Q$ is the total flow per unit time; $k$ is the permeability coefficient; $A$ is the cross-section area of model medium; $\left(h_{2}-h_{l}\right)$ is the head difference; $L$ is the seepage path; $i$ is the hydraulic gradient.

In order to study the fluid flow in porous media, seepage experiment was widely applied in many fields. With the development of seepage mechanics, researchers found that not all of the fluid flow phenomena are following with Darcy's law, but only to meet certain conditions that it can be established. More and more researches found [7 - 11] that there were many fluid flow phenomena in the fields of oil field development and water conservancy project etc. which did not follow the Darcy's law, and that were called non-Darcy's seepage. Non-Darcy's seepage was widely existed in various engineering fields, and the effect on some practical projects caused by non-Darcy's seepage can't be ignored. 
Non-Darcy's seepage of low permeability rocks existed in the oil and gas field development, and this phenomenon is very common. During the research of low permeability non-Darcy's seepage, researchers carried out the numerical calculation of CT scanning model reconstruction $[12,13]$ and the seepage experiment study of microscopic simulation model [14 - 16]. Microscopic simulation of seepage experiment has been widely applied in the research of water flooding oil and microscopic residual oil distribution. The difficulty and emphasis of the experiment are the model manufacturing and experimental observation. The core casting sheet was used as the prototype to manufacture experiment model, then through the laser etching technique or chemical corrosion to engrave the pore structure on the glass sheet, and then use the microscope to carry out the observation of seepage experiment.

The core casting sheet is shown in Fig. (2A), and the glass sheet model under microscope in Fig. (2B). The microscopic simulation model experiment can simulate the natural core well from the perspective of shape, size and pores' structure, and the definition of observation is relatively high. But the requirement of the manufacture accuracy for observation model is relatively high. The experimental devices and environment have large limitations to the experiment as well, besides it can only realize $2 \mathrm{D}$ observation.

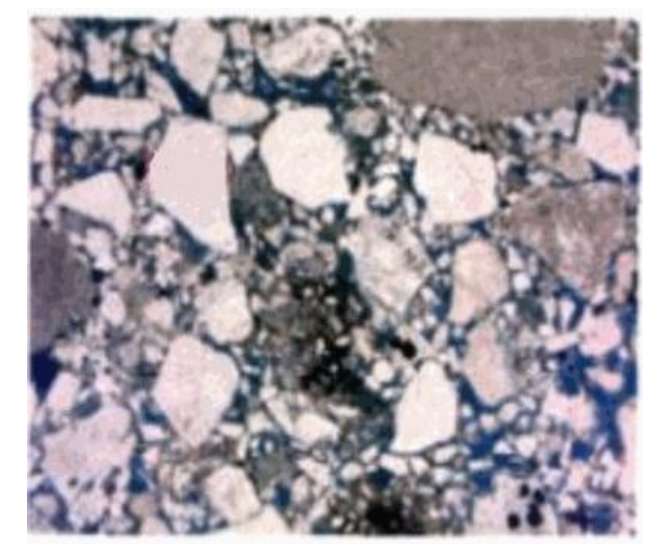

(a)

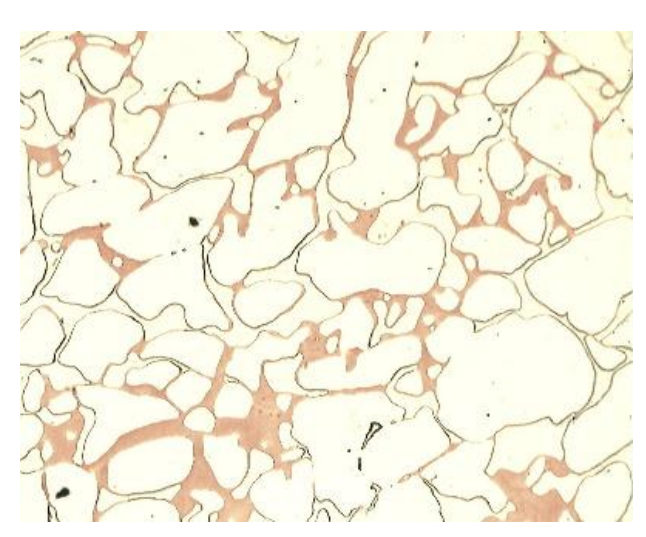

(b)

Fig. (2). (a) casting sheet of core; (b) model of glass sheet $[15,16]$.

The non-Darcy's seepage phenomena existed in water conservancy projects is generally high-speed non-Darcy's problem in rock-fill body and rupture rock mass [17]. The experimental equipment for the study on high-speed nonDarcy's seepage problem is generally large, and there are also many environment influence factors to the experimental process, therefore it is generally difficult to carry out.

In summary, the traditional seepage experiment had laid the important foundation for the development of seepage mechanics. However, with further research, the limitations of traditional experiment have greatly restricted the further development of seepage mechanics. Therefore, carrying out the research on visual seepage experiment based on TRSM has great application and theoretical significance.

\section{Study of the TRSM}

The TRSM is one of the synthetic transparent similar materials which is similar to natural rock-soil mass from the perspective of strength and deformation characteristics [18]. It is synthesized by two or more than two materials which have the similar refractive index (RI), thus it shows a better transparency. In the existing researches, amorphous silica powder and silica gel were most used as aggregate to synthesize TRSM, and the pore fluid were mineral oil and calcium bromide solution [19].

The preparation principle of TRSM is simple, and the access to get the raw materials is easy, besides the preparation price is also relatively lower [20,21]. The schematic diagram of preparation procedure is shown in Fig. (3), and the Fig. (4) is the sample of TRSM. Due to the various advantages, the TRSM shows great potential of application in the visual research for physical model test. 


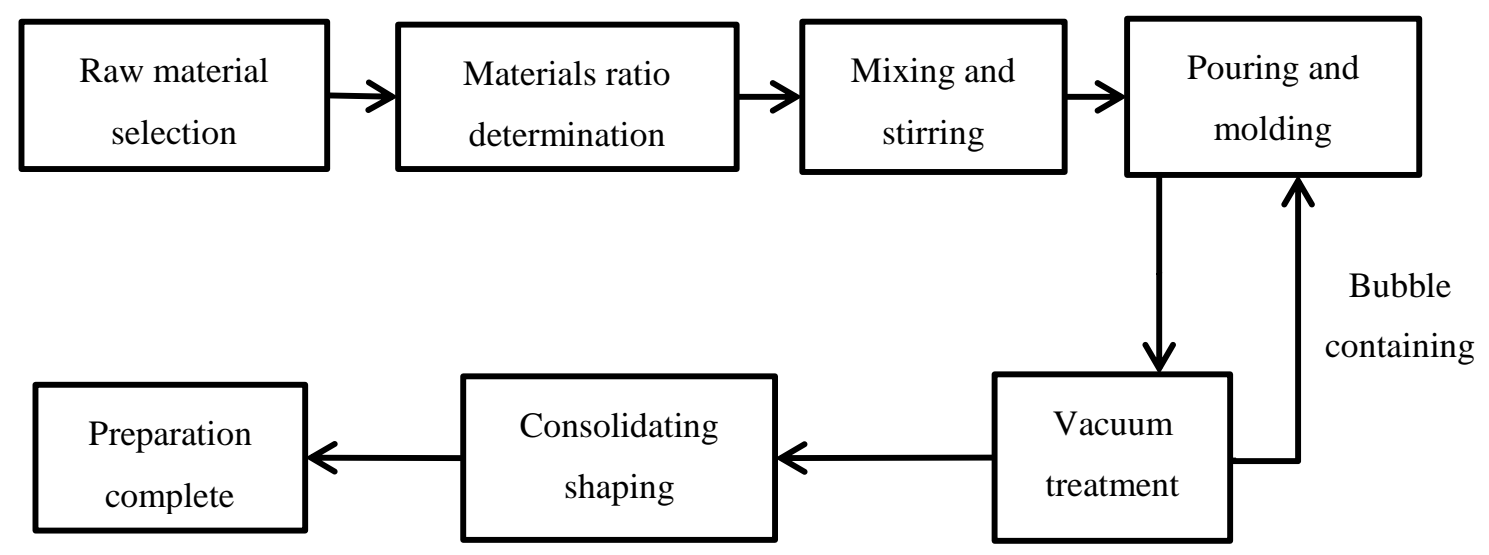

Fig. (3). Preparation procedure of TRSM.

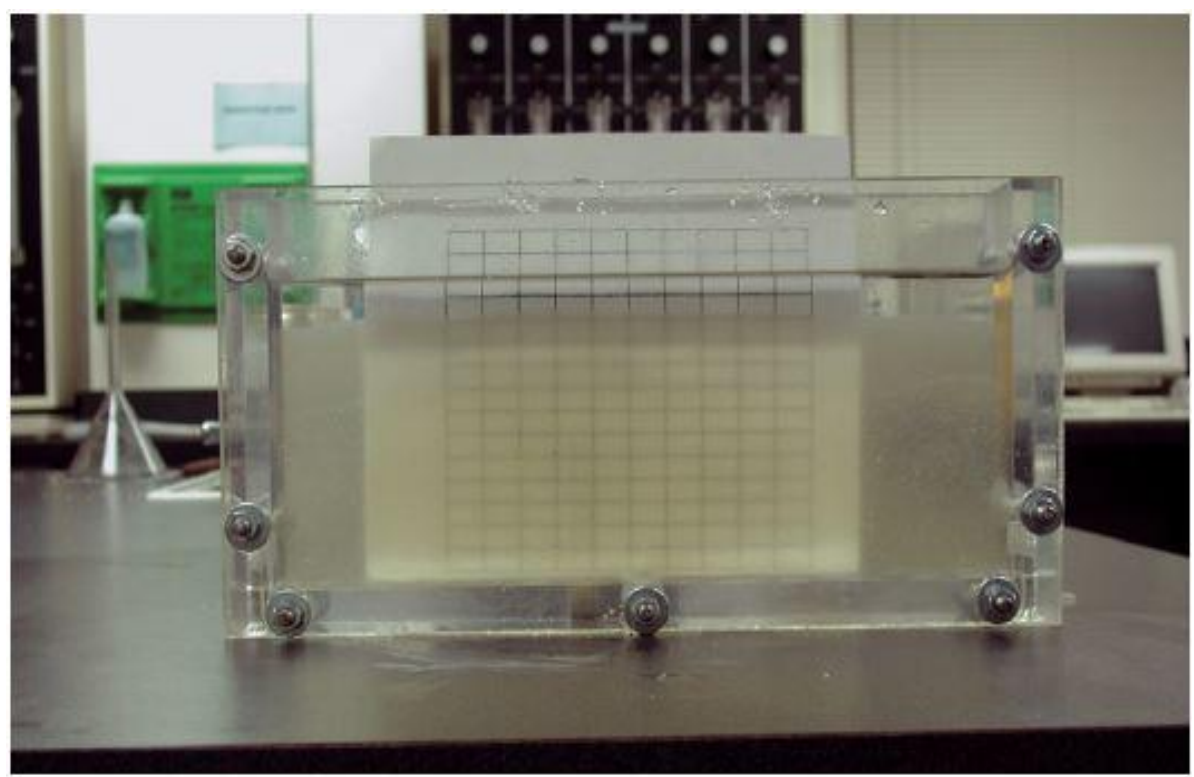

Fig. (4). Sample of TRSM [18].

The earliest research on transparent similar materials can be traced back to 1982 [22]. Allersma studied the stress and strain distribution under single shear with the model made by broken glass powder. However, due to the strength and deformation characteristics are different from the natural soil, which the model can't simulate the real situation very well. Since 1990, in order to study the flow problem of non-Newtonian fluid, Mannheimer invented a kind of transparent slurry [23].

Because of the strength and deformation characteristics of this kind of transparent slurry's consolidation are similar to natural soil, it indicated that the research on TRSM has stepped into a new stage. With years' development and exploration, the researches on visual model test have made a great progress from the perspective of simulating the spatial-temporal evolution law of deformation and crack of deep rock-mass [24], unstable mechanism of surrounding rock caused by excavation unloading [25], the inter-reaction between rock-soil mass and structures [26].

The TRSM has shown a great potential for application on visual research in geotechnical field. However, the research on seepage mechanics with TRSM is still a little literature available.

With the TRSM, visual researches can be carried out from the perspective of simulating the infiltration-flow law of liquid in rock-soil mass [27], the flow test to simulate the flow state of fluid [28], the diffusion mechanism of chemical grouting [29] and contaminant transport in porous media [30] etc. It indicates that the TRSM has a wide application prospect in seepage mechanics. 


\section{Design of Seepage Experiment Based on the TRSM}

Carrying out seepage experiment with TRSM can realize the visual observation of seepage process due to the material not only has a similar properties with natural soil from the perspective of strength and deformation, but also the hydraulic property, which were reported in the existing researches [28, 30, 31]. This kind of material can simulate the fluid flow in different medium based on the different raw material used in the preparation of transparent rock-soil material.

The biggest difference between the seepage experiment based on TRSM and traditional experiment is the model medium used in experiment. The former is transparent, while the latter is opaque. Due to the transparency property of model medium, it determinates the use of optical observation system to capture the image of seepage process during the experiment, at the same time we need utilize the imaging processing technique to manipulate image for helping to analyze the seepage law. The schematic diagram of seepage experiment device based on TRSM is shown in Fig. (5).

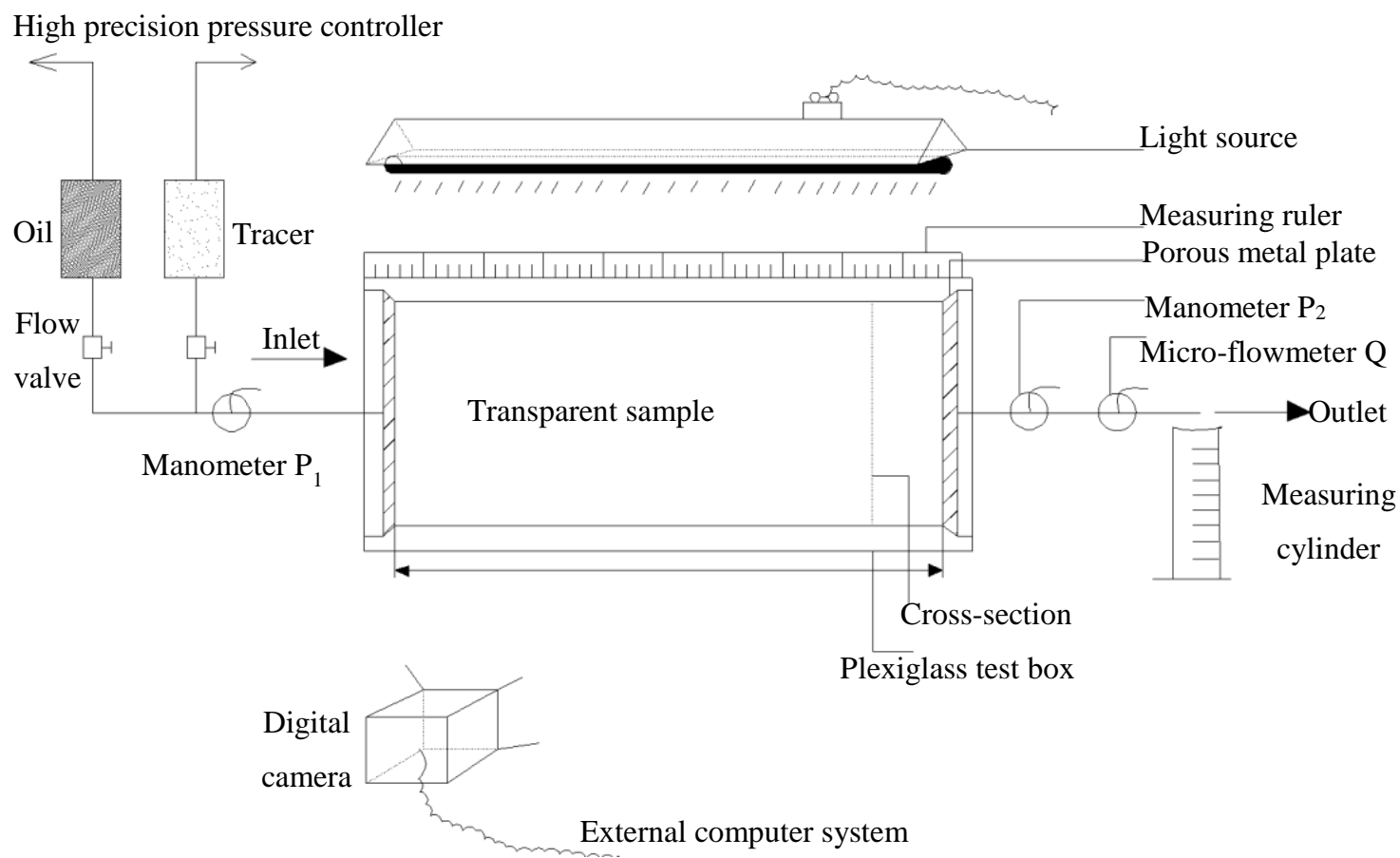

Fig. (5). Schematic diagram of seepage experiment device.

The device can provide stable and continuous differential pressure by connecting exterior constant pressure controller and high-precision flux controller valve to control the injection of fluid and tracer. The whole plexiglass experimental box is illuminated under the exterior light source, and the digital camera is utilized to capture image during the seepage experiment, then the image is loaded into an external computer system for image processing. A high-precision measuring ruler is set at the upper boundary of experimental box to measure the movement of fluid front. Manometers are set at inflow side and outflow side, a high-precision micro-flowmeter is set at outflow side, and they are all connected to computer system to realize the automatic collection of experimental data during the experiment. The required time should be recorded when the manometers achieve stability, and the flux should also be recorded when the experiment achieves steady seepage during experiment.

Then we can utilize the differential pressure flux method to calculate the seepage curve [32]. If necessary, the load system could be installed on the upper surface of experimental box, embedded senor into the sample, so as to realize the fluid-solid coupling analysis of seepage. Therefore, the equipment can be utilized to study the change of seepage law caused by the change of stress state under the external load, as well as the change of stress state caused by the fluid flow.

By utilization of the equipment, we can carry out simple 2D seepage experiment to observe the flow and diffusion law. At the same time, we can synthesize the sample with known pore structure according to the pore structure data of 
natural core. Then by using the sample with known pore structure, we can carry out quantitative experiment of waterflooding oil, two phase flow and the microscopic residual oil distribution etc. During the water-flooding oil experiment, we can intuitively observe the formation and development of fingering phenomenon, the movement and diffusion of water-flooding front could also be observed.

Through the observation of confined gas formation during the two phase flow experiment, we can form a certain qualitative understanding of the formation mechanism of the confined gas. By changing the installation position and angle of the digital camera, we can capture slice images of the different region in the sample, and the 3D seepage field analysis can be carried out through the overlap of multi-group slice images, as shown in Fig. (6), or by arrangement of multi-group digital camera to achieve the 3D analysis.

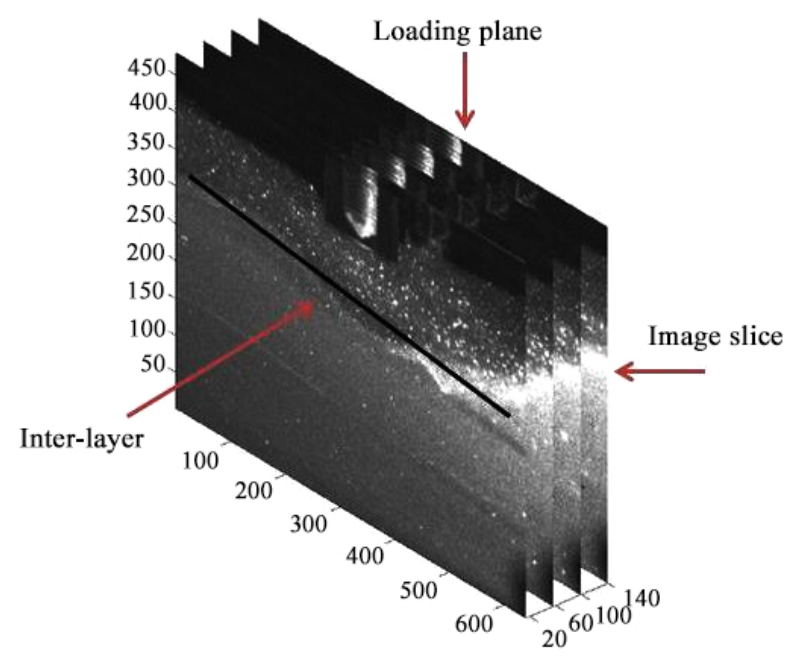

Fig. (6). 3D analysis by overlapping multi-group slice images [18].

Compared with the traditional seepage experiment, the biggest advantage of this equipment is realizing the visual observation of seepage process. The flow law, diffusion shape and occurrence state of fluid can be recorded intuitively during experiment. Compared with the microscopic model seepage experiment, the experimental equipment is more simple, and the operation is easier, besides it can also realize the $3 \mathrm{D}$ analysis of test results.

\section{CONCLUSION}

Through the comparative analysis between the traditional seepage experiment (macroscopic and microscopic) and the seepage experiment based on TRSM, following conclusions could be obtained:

1. Compared with the traditional seepage experiment, the seepage experiment based on TRSM can not only realize the visual observation of the seepage process, but also the equipment is more simple, operation relatively easier, thus it is convenience to experimental operation and theoretical teaching.

2. The seepage experiment equipment based on TRSM was introduced in detail, and the theoretical foundation was established for carrying out the subsequent seepage model experiment.

\section{ABBREVIATION LIST}

$\begin{array}{lll}\text { TRSM } & = & \text { Transparent rock-soil material } \\ \text { CT } & = & \text { Computed tomography } \\ \text { RI } & = & \text { Refractive index } \\ \text { 2D } & = & \text { 3D-two dimension, three dimension }\end{array}$

\section{CONSENT FOR PUBLICATION}

Not applicable. 


\section{CONFLICT OF INTEREST}

The authors declare that there is no conflict of interest regarding the publication of this manuscript.

\section{ACKNOWLEDGEMENTS}

This paper is financially supported by Natural Science Foundation of China (Grant No.51174170), National Science and Technology Major Project of China (Grant No. 2017ZX05037-001 and 2017ZX05013-001).

\section{REFERENCES}

[1] N.I. Ge, The Modern Mechanics of Fluids Flow in Oil Reservoir., Petroleum Industry Press: Beijing, 2011.

[2] S.P. Guo, C.Q. Liu, and Y.Z. Huang, "The recent developments of flow through porous media", Adv. Mech., vol. 16, pp. 441-452, 1986.

[3] J.L. Liu, Y.W. Liu, and Y.Z. Huang, "Retrospect and prospect of flow through porous media", Mech. Prac., vol. 30, pp. 94-97, 2008.

[4] J.F. Zhou, "Mechanics of porous media flow: Status and perspectives", Mech. Prac., vol. 29, pp. 1-6, 2007.

[5] Z.Q. Chen, and B.Y. Yu, "Research progress of seepage mechanics in rock mass affected by mining", J. Southwest Petro. Uni. (Sci. and Tech. Edition), vol. 37, pp. 69-76, 2015.

[6] A.E. Scheidegger, The Physics of Flow in Porous Media., University of Toronto Press: Toronto, 1974.

[7] J.J. Liu, X.G. Liu, and Y.R. Hu, "Study on nonlinear seepage of rock of low permeability", Chi. J. Rock Mech. Eng., vol. 22, pp. 556-561, 2003

[8] E.Z. Wang, W.Z. Zhang, and X.M. Han, "Experimental study on coupled flow through low permeability rock under confining pressure", J. Tsinghua Uni. (Sci. and Tech.), vol. 45, pp. 764-767, 2005.

[9] F. Yan, M.L. Zhan, and B.Y. Su, "Model test on unsaturated seepage of dike", Chi. J. Geotech. Eng., vol. 26, pp. 296-298, 2004.

[10] F. Qin, and Y. Wang, "“Research advance of non-darcy flow”, J. of Chi. Three Gorges Uni", Nat. Sci., vol. 31, pp. 25-29, 2009.

[11] X.D. Qiu, Z.L. Yan, and B.J. Yao, "Effect of particle-size characteristic on the seepage property of rock-fill", J. Sichuan Uni. (Eng. Sci. Edition), vol. 35, pp. 06-09, 2003.

[12] M. Pollefeys, and R.D. Nistér, "Detailed real-time urban 3D reconstruction from video", Int. J. Comput. Vis., vol. 78, pp. 143-167, 2008. [http://dx.doi.org/10.1007/s11263-007-0086-4]

[13] R. Song, J. Liu, and M. Cui, "Single- and two-phase flow simulation based on equivalent pore network extracted from micro-CT images of sandstone core", Springerplus, vol. 5, no. 1, p. 817, 2016. [http://dx.doi.org/10.1186/s40064-016-2424-x] [PMID: 27390657]

[14] D.W. Li, L.H. Zhang, and K.M. Zhou, "Gas-water two-phase flow mechanism in visual microscopic pore model", J. Chi. Uni. Petr. (Natural Sci. Edition), vol. 32, no. 80, p. 83, 2008.

[15] K. Yang, and S.Y. Xu, "Experiment methods of microcosmic remaining oil", Fault-Block Oil \& Gas Field, vol. 16, pp. 75-77, 2009.

[16] Y.J. Yan, J.Y. Chen, and J.S. Guo, "A visualized experiment on gas-water two-phase seepage through oolitic reservoirs in the Longgang Gas Field, Sichuan Basin", Nat Gas Ind., vol. 32, pp. 64-66, 2012.

[17] Y.S. Li, X.F. Li, and S.N. Teng, "Effect of Non-Darcy Flow Moving Boundary of High Velocity Nearby Wellbore on Productivity Prediction", J. of Southwest Petr. Uni. (Sci.\& Tech. Edition), vol. 37, pp. 128-134, 2015.

[18] M. Iskander, Modeling with Transparent Soils., Springer Berlin Heidelberg: Berlin, 2010. [http://dx.doi.org/10.1007/978-3-642-02501-3]

[19] M. Iskander, R.J. Bathurst, and M. Omidvar, "Past, present and future of transparent soils", Geo. Testing J., vol. 38, pp. 557-573, 2015.

[20] M. Iskander, J.Y. Liu, and S. Sadek, "Transparent amorphous silica to model clay", J. Geo. Geo. Eng., vol. 128, pp. 262-273, 2002. [http://dx.doi.org/10.1061/(ASCE)1090-0241(2002)128:3(262)]

[21] M. Iskander, S. Sadek, and J.Y. Liu, "“Optical measurement of deformation using transparent silica gel to model sand”, Int", J. Physical Model in Geomechanics, vol. 2, pp. 13-26, 2002.

[22] H.G. Allersma, "Photo-elastic stress analysis and strains in simple shear", IUTAM Conference, 1982 pp. 345-353

[23] R. Mannheimer, "Slurries you can see through", Tech. Today, vol. 3, p. 2, 1990.

[24] G.A. Xu, "Research on mechanism and evolution law of rock deformation and cracking around deep tunnels", Ph.D. thesis, China University of Mining and Technology, Xuzhou, ON, China, 2011.

[25] Y.H. Li, Z.B. Lin, and X.L. Qin, "Study of development of transparent rock mass for physical similar experiment and its mechanical properties", J. Chi. Uni. Min. Tech., vol. 44, pp. 977-982, 2015.

[26] G.Q. Kong, L.D. Zhou, and Z.T. Wang, "Shear modulus and damping ratios of transparent soil manufactured by fused quartz", Mater. Lett., vol. 182, pp. 257-259, 2016. [http://dx.doi.org/10.1016/j.matlet.2016.07.012] 
[27] G.A. Siemens, S.B. Peters, and W.A. Take, "Comparison of confined and unconfined infiltration in transparent porous media", Water Res. Rese., vol. 49, pp. 851-863, 2013. [http://dx.doi.org/10.1002/wrcr.20101]

[28] J.Y. Liu, Visualization of 3D deformations using transparent "soil” models", Ph.D. thesis, Polytechnic University, New York, ON, USA, 2003.

[29] J.Y. Liu, W.H. Sui, and Y. Gao, "Visualization of Chemical Grout Permeation in Transparent Soil", Geo. Testing J., vol. 38, pp. 2477-2488, 2015 .

[30] R.F. Serrano, M. Iskander, and K. Tabe, "3D contaminant flow imaging in transparent granular porous media", Geo. Lett., vol. 1, pp. 71-78, 2011

[31] J.Y. Liu, M. Iskander, and S. Sadek, "Consolidation and permeability of transparent amorphous silica", Geo. Testing J., vol. 26, pp. 390-401, 2003.

[32] C.G. Xue, Q. He, and Z.M. Yang, "Experimental study of the nonlinear seepage with two-dimensional physical model", J. Southwest Petr. Uni. (Sci. and Tech. Edition), vol. 33, pp. 101-104, 2011.

\section{(C) 2017 Liu et al.}

This is an open access article distributed under the terms of the Creative Commons Attribution 4.0 International Public License (CC-BY 4.0), a copy of which is available at: https://creativecommons.org/licenses/by/4.0/legalcode. This license permits unrestricted use, distribution, and reproduction in any medium, provided the original author and source are credited. 